Original Research Paper

\title{
Dynamics and Stability of $q$-Fractional Order Pantograph Equations With Nonlocal Condition
}

\author{
${ }^{1}$ Vivek Devaraj, ${ }^{2}$ Kuppusamy Kanagarajan and ${ }^{3}$ Seenith Sivasundaram \\ ${ }^{1,2}$ Department of Mathematics, Sri Ramakrishna Mission Vidyalaya \\ College of Arts and Science,Coimbatore-641020, India \\ ${ }^{3}$ Department of Mathematics, College of Engineering, Daytona Beach, FL 32114, USA
}

Article history

Received: 1-12-2017

Revised: $13-12-2017$

Accepted: 2-02-2018

Corresponding Author:

Seenith Sivasundaram

Department of Mathematics,

Daytona Beach, FL 32114, USA

Email: seenithi@gmail.com

\begin{abstract}
This paper deals with some existence and Ulam-Hyers- $q$ Mittag-Leffler stability results for $q$-fractional order pantograph equation. An application is made of a Darbo's fixed point theorem for the existence of solutions.
\end{abstract}

Keywords: Fractional $q$-Fractional Order Pantograph Equation, Nonlocal Condition, Existence, Fixed Point, Ulam-Hyers- $q$-Mittag-Leffler Stability

\section{Introduction}

Early works about $q$-difference calculus or quantum calculus initially read in Jackson $(1908 ; 1910)$. The difference equations are widely used in mathematical physical problems, sampling theory of signal analysis, dynamical system and quantum models and heat and wave equations. Recently, some researchers have noticed their attention to discrimination research of the fractional $q$-difference calculus, we refer readers to (Agarwal et al., 2014; Liang and Zhang, 2012; Al-Yami, 2016; Stankovic et al., 2009). For a long time, in many areas the fractional differential equations are very popular. For example, engineers and scientists have developed new methods that include fractional equations; we refer to (Hilfer, 1999; Podlubny, 1999). Since the beginning of the last decade, the fractional $q$-differential equations have become an important mathematical tool.

The pantograph equations have been widely studied (Balachandran et al., 2013; Liu and Li, 2004) and references therein)since they can be utilized to depict many phenomena that arise in electro dynamics, probability, quantum mechanics, dynamical systems and number theory. Recently, fractional pantograph differential equations have been considered by many researchers. One of the motivating topics in this area is the research of the existence of solutions by fixed point theorems, we refer to (Balachandran et al., 2013).

The Ulam stability of functional equation, which was Ulam founded for a speech to a conference at the University of Wisconsin in 1940, is one of the important subjects in the mathematical analysis area. The finding of Ulam stability plays a vital role in regard to this subject. For detailed study on the progress of Ulam type (U-H) stability, readers refer to (Andras and Kolumban, 2013; Jung, 2004; Muniyappan and Rajan, 2015) and the references therein. The credit of solving this problem partially goes to Hyers. To study U-H stability of fractional differential equations, different researchers studied their works with different methods, see (Ibrahim, 2012; Wang et al., 2011; Wang and Zhou, 2012; Wang and Zhang, 2014). Koca (2015) proved local asymptotics stability of $q$-fractional nonlinear dynamics systems. Inspired by the above discussion, we initiate the existence and U-H- $q$-Mittag-Leffler stability for $q$ fractional pantograph equations.

Consider the following system represented by the $q$ fractional order pantograph equation with nonlocal condition of the form:

$$
\begin{aligned}
& { }^{c} D_{q}^{\alpha} x(t)=F(t, x(t), x(\lambda t)), t \in \quad \quad:=[0, T] \\
& x(0)+g(x)=x_{0}
\end{aligned}
$$

where, ${ }^{c} D_{q}^{\alpha}$ is the Caputo fractional $q$-derivative, $q \in$ $(0,1)$. Let $0<\alpha<1,0<\lambda<1$ and $F: f \times \mathfrak{x} \times \mathfrak{x} \rightarrow \mathfrak{x}, \mathrm{g}: \mathscr{C}(f, \mathfrak{x}) \rightarrow \mathfrak{x}$ are given continuous functions.

Observing that system (1) is equivalent to the following nonlinear integral equation:

$$
\begin{aligned}
& x(t)=x_{0}-g(x) \\
& +\frac{1}{\Gamma_{q}(\alpha)} \int_{0}^{(t-q s)^{(\alpha-1)}} F(s, x(s), x(\lambda s)) d_{q} s
\end{aligned}
$$


Let $\mathscr{C}(\mathscr{F}, \mathscr{X})$ be the Banach space of continuous function $x(t)$ with $x(t) \in \mathscr{X}$ for $t \in \mathscr{J}$ and $\|x\| \mathscr{C}(\mathscr{F}, \mathcal{X})=$ $\max _{t} \in \mathscr{f}\|x(t)\|$.

In passing, we note that the application of nonlinear condition $x(0)+g(x)=x_{0}$ in physical problems yeilds better effect than the initial condition $x(0)=x_{0}$ (Bashir and Sivasundaram, 2008).

The outline of the paper is as follows. In section 2 , we give some basic definitions and results con-cerning the fractional $q$-calculus. In section 3, we present our main results by fixed point theorems. Stability analysis is discussed in section 4 .

\section{Prerequisites}

For detailed study on $q$-fractional calculus, one can refer to (Al-Yami, 2016; Stankovic et al., 2009).

Let $q \in(0,1)$ and define:

$$
[a]_{q}=\frac{q^{a}-1}{q-1}=q^{a-1}+\cdots+1, a \in \mathbb{R}
$$

The $q$-analogue of the Pochhammer symbol was presented as follow:

$$
(a ; q)_{0}=1,(a ; q)_{n}=\prod_{k=0}^{n-1}\left(1-a q^{k}\right), a \in \mathbb{R}, n \in \mathbb{N} \cup\{\infty\}
$$

In general, if $\alpha \in \mathbb{R}$ thereafter:

$$
(a ; q)_{\infty}=\prod_{i=0}^{\infty}\left(1-a q^{i}\right),(a ; q)_{\alpha} \frac{(a ; q)_{\infty}}{\left(a q^{\alpha} ; q\right)_{\infty}}
$$

The $q$-gamma function is defined by:

$$
\Gamma_{q}(x)=(q ; q)_{x-1}(1-q)^{1-x}, x \in \mathbb{R} \mid\{0,-1,-2, \ldots\}, 0<q<1
$$

and satisfies $\Gamma_{q}(x+1)=[x]_{q} \Gamma_{q}(x)$.

The $q$-derivative of a function $\mathrm{F}(x)$ is here defined by:

$$
D_{q} F(x)=\frac{d_{q} F(x)}{d_{q} x}=\frac{F(q x)-F(x)}{(q-1) x}
$$

and:

$$
D_{q}^{n} F(x)=\left\{\begin{array}{cc}
F(x) & \text { if } n=0 \\
D_{q} D_{q}^{n-1} F(x) & \text { if } n \in \mathbb{N}
\end{array}\right.
$$

The $q$-integral of a function $\mathrm{F}$ defined in the interval $[0, T]$ is provided by:

$$
\int_{0}^{x} F(t) d_{q} t=x(1-q) \sum_{n=0}^{\infty} F\left(x q^{n}\right) q^{n}, 0 \leq|q|<1, \quad x \in[0, b]
$$

now, it can be defined an operator $I_{q}^{n}$, as follows:

$$
\left(I_{q}^{0} F\right)(x)=F(x) \text { and }\left(I_{q}^{n} F\right)(x)=I_{q}\left(I_{q}^{n-1} F\right)(x), n \in N
$$

We can point the basic formula which will be used at a later time:

$$
{ }_{s} D_{q} t^{\alpha}(s / t ; q)_{\alpha}=-[\alpha]_{q} t^{\alpha-1}(q s / t ; q)_{\alpha-1}
$$

where, ${ }_{s} D_{q}$ denotes the $q$-derivative with respect to variable $s$.

Definition 2.1. (Al-Yami, 2016)

Let $\alpha \leq 0$ and $F$ be a function defined on $[0, T]$. The fractional $q$-integral of the Riemann-liouville type is $\left(I_{q}^{0} F\right)(x)=F(x)$ and:

$$
\left(I_{q}^{\alpha} F\right)(x)=\frac{x^{\alpha-1}}{\Gamma_{q}(\alpha)} \int_{0}^{x}(q t / x ; q)_{\alpha-1} F(t) d_{q} t, \quad \alpha \in \mathbb{R}^{+}, x \in[0, T]
$$

\section{Definition 2.2. (Al-Yami, 2016)}

The fractional $q$-derivative of the Caputo type of order $\alpha>0$ is defined by:

$$
\left({ }_{c} D_{q}^{\alpha} F\right)(x)=\left(I_{q}^{[\alpha]-\alpha} D_{q}^{[\alpha]} F\right)(x)
$$

where, $[\alpha]$ is the smallest integer greater than or equal to $\alpha$.

\section{Definition 2.3}

The fractional q-derivative of the Riemann-Lioville type of order $\alpha \geq 0$ is defined by $D_{q}^{0} f(x)=f(x)$ and:

$$
\left(D_{q}^{0} F\right)(x)=\left(D_{q}^{\lceil\alpha\rceil} I_{q}^{m-\lceil\alpha\rceil} F\right)(x) \alpha>0
$$

\section{Lemma 2.4. (Al-Yami, 2016)}

Let $x>0$ and $\alpha \in \mathbb{R}^{+} / \mathbb{N}$. Then, the following equality holds:

$$
\left(I_{q}^{\alpha} \quad D_{q}^{\alpha} F\right)(x)=F(x)-\sum_{k=0}^{\lceil\alpha\rceil} \frac{x^{k}}{\Gamma_{q}(k+1)}\left(D_{q}^{k} F\right)(0)
$$

\section{Denition 2.5. (Hassan, 2016)}

The $q$-Mittag-Leffler-function defined as:

$$
e_{\alpha, \mu}(z ; q)=\sum_{k=0}^{\infty} \frac{z^{k}}{\Gamma_{q}(\alpha k+\mu)},|z|<(1-q)^{\alpha}
$$


when $\mu=1$ we simply use $e_{\alpha}(z ; q):=e_{\alpha, 1}(z ; q)$.

\section{Remark 2.6}

The $q$-Mittag-Leffler function will tend to the classical one when $q \rightarrow 1$.

\section{Theorem 2.7. (Darbo's Fixed Point Theorem} (Lakshmikantham, 1994),p.no.21)

Let $K$ be a bounded, closed convex set of a Banach space $\mathscr{X}$. Suppose that $T$ and $S$ are two mappings from $K$ to $\mathscr{X}$ satisfying:

- $T x+S y \in K$ for any $x, y \in K$

- $\quad T$ is a contraction mapping

- $\quad S$ is a completely continuous on $K$

Then $T+S$ has atleast a fixxed point on $K$.

\section{Main Results}

Let us list some hypotheses to prove our existence results:

(A1) $\mathrm{F}: \mathscr{f} \times \mathscr{X} \times \mathscr{X} \rightarrow \mathscr{X}$ is continuous function

(A2) There exists a positive constant $L>0$ such that:

$$
|F(t, x, u)-F(t, y, v)| \leq L(|x-y|+|u-v|)
$$

for $t \in \mathscr{f}, u, v, x, y \in \mathscr{X}$

(A3) $g: \mathscr{C}(\mathscr{C}, \mathscr{X}) \rightarrow \mathscr{X}$ is continuous function and there exists a constant $b>0$, such that:

$$
|g(x)-g(y)| \leq b|x-y|, \text { for all } x, y \in \mathscr{C}(\mathscr{f}, \mathfrak{X})
$$

(A4) There exists a function $\mu \in L^{1}(\varnothing$ such that:

$$
|F(t, x, y)| \leq \mu(t) \text { for all } t \in \quad, x, y \in \mathscr{X}
$$

We are now ready to present our results. The existence results are based on Darbo's fixed point theorem.

\section{Theorem 3.1}

Assume $(A 1),(A 3)$ with $b<1$ and $(A 4)$ hold. Then, system (1) has at least one fixed point on $\mathscr{f}$.

\section{Proof}

Let $P$ and $Q$ the two operators defined on $B_{r}$ by:

$$
\begin{aligned}
& (P x)(t):=\frac{t^{\alpha-1}}{\Gamma_{q}(\alpha)} \int_{0}^{t}(q s / t ; q)_{\alpha-1} F(s, x(s), x(\lambda s)) d_{q} s, \\
& (Q x)(t):=x_{0}-g(x)
\end{aligned}
$$

respectively. Note that if $x, y \in B_{r}$, where, $B_{r}:=\{x \in$ $(\mathscr{f}, \mathfrak{X}):|x| \leq r\}$.

Set $G=\max _{x \in \mathscr{E}}(\mathscr{f} \mathfrak{x})|g(x)|$, then $\mathrm{P} x+\mathrm{Q} y \in B_{r}$.
Indeed it is easy to check the inequality:

$$
\begin{aligned}
|P x+Q y| & =\left|x_{0}-g(y)+\frac{t^{\alpha-1}}{\Gamma_{q}(\alpha)} \int_{0}(q s / t ; q)_{\alpha-1} F(s, x(s), x(\lambda s)) d_{q} s\right| \\
& \leq\left|x_{0}\right|+|g(y)|+\frac{t^{\alpha-1}}{\Gamma_{q}(\alpha)} \\
& \int_{0}\left|(q s / t ; q)_{\alpha-1} F(s, x(s), x(\lambda s))\right| d_{q} s \\
& \leq\left|x_{0}\right|+G+\frac{|\mu| t^{\alpha-1}}{\Gamma_{q}(\alpha)} \int_{0}(q s / t ; q)_{\alpha-1} d_{q} s \\
& \leq\left\|x_{0}\right\|+G+\frac{\|\mu\|_{L^{1}} T^{\alpha}}{\Gamma_{q}(\alpha+1)} \leq r
\end{aligned}
$$

Thus:

$$
P x+Q y \in B_{r}
$$

By (A3), it is also clear that $Q$ is a contraction mapping. Produced from continuity of $x$, the operator $(P x)(t)$ is continuous in accordance with $(A 1)$. Also we observe that:

$$
\begin{aligned}
|(P x)(t)| & \leq \frac{t^{\alpha-1}}{\Gamma_{q}(\alpha)} \int_{0}^{t}(q s / t ; q)_{\alpha-1}|F(s, x(s), x(\lambda s))| d_{q} s \\
& \leq \frac{\|\mu\|_{L^{1}} T^{\alpha}}{\Gamma_{q}(\alpha+1)}
\end{aligned}
$$

Then $\mathrm{P}$ is uniformly bounded on $B_{r}$.

Now let's prove that $(P x)(t)$ is equicontinuous.

Let $t_{1}, t_{2} \in J, t_{2} \leq t_{1}$ and $x \in B_{r}$. Using the fact $F$ is bounded on the compact set $\mathscr{f} \times B_{r}$ (thus $\max _{(\mathrm{t}, x, y) \in \mathscr{f} \times}$ $\left.{ }_{B r}|F(t, x, y)|:=C_{0}<\infty\right)$.

We will get:

$$
\begin{aligned}
& \left|(P x)\left(t_{1}\right)-(P x)\left(t_{2}\right)\right| \\
& =\mid \begin{array}{l}
\frac{t_{1}^{\alpha-1}}{\Gamma_{q}(\alpha)} \int_{0}^{t_{1}}\left(q s / t_{1} ; q\right)_{\alpha-1} F(s, x(s), x(\lambda s)) d_{q} s \\
-\frac{t_{2}^{\alpha-1}}{\Gamma_{q}(\alpha)} \int_{0}^{t_{2}}\left(q s / t_{2} ; q\right)_{\alpha-1} F(s, x(s), x(\lambda s)) d_{q} s \mid
\end{array} \\
& \leq \frac{1}{\Gamma_{q}(\alpha)}\left[\begin{array}{l}
t_{1}^{\alpha-1} \int_{t_{2}}^{\mu_{1}}\left(q s / t_{1} ; q\right)_{\alpha-1}|F(s, x(s), x(\lambda s))| d_{q} s \\
+\int_{0}^{r_{2}}\left(t_{2}^{\alpha-1}\left(q s / t_{2} ; q\right)_{\alpha-1}-t_{1}^{\alpha-1}\left(q s / t_{1} ; q\right)_{\alpha-1}\right) \\
|F(s, x(s), x(\lambda s))| d_{q} s
\end{array}\right] \\
& \leq \frac{C_{0}}{\Gamma_{q}(\alpha)}\left[\begin{array}{l}
t_{1}^{\alpha-1} \int_{t_{2}}^{r_{1}}\left(q s / t_{1} ; q\right)_{\alpha-1} d_{q} s \\
\left.+\int_{0}^{t_{2}}\left(t_{2}^{\alpha-1}\left(q s / t_{2} ; q\right)_{\alpha-1}-t_{1}^{\alpha-1}\left(q s / t_{1} ; q\right)_{\alpha-1}\right) d_{q} s\right]
\end{array}\right.
\end{aligned}
$$


which is autonomous of $x$ and head for zero as $t_{1}-t_{2}$ $\rightarrow 0$ consequently $P$ is equicontinuous. Thus, $P$ is relatively compact on $B_{r}$. By the Arzela-Ascoli theorem, $\mathrm{P}$ is compact. We now conclude the result of the theorem based on the Darbo's fixed point. Thus, the problem (1) has at least one fixed point on $\mathscr{F}$.

\section{Stability Analysis}

In this section, we define some basic concepts of UH- $q$-Mittag-Leffler stability. We adopt some ideas in (Otrocol and Ilea, 2013).

\section{Definition 4.1}

The Equation (1) is $U-H$ - $q$-Mittag-Leffler stable with respect to $e_{\alpha}\left(t^{\alpha} ; q\right)$ if there exists $C_{e_{\alpha}}$ such that for each $\epsilon$ $>0$ and for each solution $z \in \mathscr{E}(\mathscr{F} ; \mathfrak{X})$ of the inequality:

$$
\left|{ }^{c} D_{q}^{\alpha} z(t)-F(t, z,(t), z(\lambda t))\right| \leq \epsilon e_{\alpha}\left(t^{\alpha} ; q\right), t \in \mathscr{J}
$$

There exists a solution $x \in \mathscr{C}(\mathscr{f}, \mathscr{X})$ of Equation (1) with:

$$
|z(t)-x(t)| \leq C e_{\alpha} \epsilon e_{\alpha}\left(t^{\alpha} ; q\right), \quad t \in \mathscr{J}
$$

where, $e_{\alpha}\left(t^{\alpha} ; q\right)$ is the $q$-Mittag-Leffler function.

\section{Remark 4.2}

A function $z \in \mathscr{C}(\mathscr{C}, \mathscr{X})$ is a solution of the inequality:

$$
\left|{ }^{c} D_{q}^{\alpha} z(t)-F(t, z(t), z(\lambda t))\right| \leq \epsilon e_{\alpha}\left(t^{\alpha} ; q\right), \quad t \in \mathscr{\ell}
$$

if and only if there exists a function $h \in \mathscr{C}(\mathscr{F}, \mathfrak{X})$ such that:

1. $|h(t)| \leq \epsilon e_{\alpha}\left(t^{\alpha} ; v\right), \quad t \in \mathscr{J}$

2. ${ }^{c} D_{q}^{\alpha} z(t)=F(t, z(t), z(\lambda t))+h(t), \quad t \in \mathcal{f}$

\section{Lemma 4.3}

If a function $z \in \mathscr{C}(\mathscr{F}, \mathscr{X})$ is a solution of the inequality:

$$
\left.\mid{ }^{C} D_{q}^{\alpha} z(t)-F(t, z)(t), z(\lambda t)\right) \mid \leq \epsilon e_{\alpha}\left(t^{\alpha} ; q\right), \quad t \in \mathcal{C}
$$

then:

$$
\left|\begin{array}{l}
z(t)-z_{0}+g(z)-\frac{1}{\Gamma_{q}(\alpha)} \\
\int_{0}^{t}(t-q s)^{(\alpha-1)} F(s, z(s), z(\lambda s)) d_{q} s
\end{array}\right| \leq \epsilon e_{\alpha}\left(t^{\alpha} ; q\right)
$$

Proof

The proof directly follows from Remark 4.2, we have:

$$
\begin{aligned}
& \left|z(t)-z_{0}+g(z)-\frac{1}{\Gamma_{q}(\alpha)} \int_{0}^{t}(t-q s)^{(\alpha-1)} F(s, z(s), z(\lambda s)) d_{q} s\right| \\
& \leq \frac{\epsilon}{\Gamma_{q}(\alpha)} \int_{0}^{t}(t-q s)^{(\alpha-1)} e_{\alpha}\left(s^{\alpha} ; q\right) d_{q} s \\
& \leq \frac{\epsilon}{\Gamma_{q}(\alpha)} \int_{0}^{t}(t-q s)^{(\alpha-1)} \sum_{k=0}^{\infty} \frac{s^{k \alpha}}{\Gamma_{q}(k \alpha+1)} d_{q} s \\
& \leq \frac{\epsilon}{\Gamma_{q}(\alpha)} \sum_{k=0}^{\infty} \frac{s^{k \alpha}}{\Gamma_{q}(k \alpha+1)} \int_{0}^{t}(t-q s)^{(\alpha-1)} s^{k \alpha} d_{q} s \\
& =\epsilon \sum_{k=0}^{\infty} \frac{t^{(k+1)} \alpha}{\left.\Gamma_{q}((k+1) \alpha+1)\right)} \\
& =\epsilon \sum_{n=0}^{\infty} \frac{t^{n \alpha}}{\Gamma_{q}(n \alpha+1)} \\
& =\epsilon e_{\alpha}\left(t^{\alpha} ; q\right)
\end{aligned}
$$

\section{Denition 4.4. (Otrocol and Ilea, 2013)}

Let $(\mathscr{X}, d)$ be a metric space. $A n A: \mathscr{X} \rightarrow \mathscr{X}$ is a Picard operator if there exists $x^{*} \in \mathscr{X}$ such that $(i) F_{A}=x^{*}$ where $F_{A}=\{x \in \mathscr{X}: A(x)=x\}$ is the fixed point set of $A$; (ii) the sequence $\left(A^{n}\left(x_{0}\right)\right)_{n \in \mathrm{N}}$ converges to $x^{*}$ for all $x_{0} \in \mathscr{X}$

\section{Lemma 4.5. (abstract Gronwall lemma (Otrocol and} Ilea, 2013))

Let $(\mathscr{X}, d, \leq)$ be an ordered metric space and $A: \mathscr{X} \rightarrow \mathscr{X}$ be an increasing Picards operator $\left(F_{A}=\left\{x_{A}^{*}\right\}\right)$. Then, for $x$ $\in \mathscr{X}, x \leq A(x)$ implies $x \geq x_{A}^{*}$.

Lemma 4.6. (Henry-Gronwall inequality (Hyers et al., 1998))

Let $y, w:[0, T) \rightarrow[0, \infty)$ be continuous function where $T \leq \infty$. If $w$ is nondecreasing and there are constants $k \geq$ 0 and $0<\alpha<1$ such that:

$$
y(t) \mid \leq w(t)+k \int_{0}^{t}(t-q s)^{(\alpha-1)} y(s) d_{q} s, \quad t \in[0, T)
$$

then:

$$
y(t) w(t)+\int_{0}^{t}\left(\sum_{0}^{t} \frac{\left(k \Gamma_{q}(\alpha)\right)^{n}}{\Gamma_{q}(n \alpha)}(t-q s)^{(n \alpha-1)} w(s)\right) d_{q} s, t \in[0, T)
$$

\section{Remark 4.7}

By the hypothesis of Lemma 4.6, let $w(t)$ be a nondecresing function on $[0, T)$. Then we have $y(t) \leq$ $w(t) e_{\alpha}\left(k \Gamma_{q}(\alpha) t^{\alpha} ; q\right)$. 
We are now in a position to state and prove our stability results for problem (1). The arguments are based on the Banach contraction principle with respect to Chebyshev norm.

\section{Theorem 4.8}

Assume that hypotheses (A1)-(A3) are fulfilled. If:

$$
b<\frac{1}{2} \text { and } L \leq \frac{\Gamma_{q}(\alpha+1)}{4 T^{\alpha}}
$$

Then the Equation (1) has a unique solution.

\section{Proof}

The operator P: $\mathscr{E}(\mathscr{C}, \mathfrak{X}) \rightarrow \mathscr{C}(\mathscr{f}, \mathfrak{X}):$

$$
\begin{aligned}
& (P x)(t)=x_{0}-g(x) \\
& +\frac{1}{\Gamma_{q}(x)} \int_{0}^{(}(t-q s)^{(\alpha-1)} F(s, x(s), x(\lambda s)) d_{q} s
\end{aligned}
$$

Choose $r \geq 2\left(\left\|x_{0}\right\|+G+\frac{M T^{q}}{\Gamma_{q}(\alpha+1)}\right)$ and let $\max _{t \in \mathcal{C}}$ $|\mathrm{F}(t, 0,0)|=M$.

Then we can show that $P B_{r} \subset B_{r}$.

So let $x \in B_{r}$ and set $G=\max _{x \in \mathscr{E}(\mathscr{F}, g)}|g(x)|$. Then we get:

$$
\begin{aligned}
& |\operatorname{Px}(t)| \\
& =\left|x_{0}-g(x)+\frac{t^{\alpha-1}}{\Gamma_{q}(\alpha)} \int_{0}(q s / t ; q)_{(\alpha-1)} F(s, x(s), x(\lambda s)) d_{q} s\right| \\
& \leq\left|x_{0}\right|+G+\frac{t^{\alpha-1}}{\Gamma_{q}(\alpha)} \int_{0}^{t}\left|(q s / t ; q)_{\alpha-1} F(s, x(s), x(\lambda s))\right| d_{q} s \\
& \leq\left|x_{0}\right|+G+\frac{t^{\alpha-1}}{\Gamma_{q}(\alpha)} \int_{0}^{t}(q s / t ; q)_{\alpha-1} \\
& \left(\max _{s \in J}|F(s, x(s), x(\lambda s))-F(s, 0,0)|+\max _{s \in J}|F(s, 0,0)|\right) d_{q} s \\
& \leq\left|x_{0}\right|+G+\frac{t^{\alpha-1}}{\Gamma_{q}(\alpha)} \int_{0}^{t}(q s / t ; q)_{\alpha-1} L[|x(s)|+|x(\lambda s)|] d_{q} s \\
& +\frac{M t^{\alpha-1}}{\Gamma_{q}(\alpha)} \int_{0}(q s / t ; q)_{\alpha-1} d_{q} s \\
& \leq\left|x_{0}\right|+G+\frac{2 L r t^{\alpha-1}}{\Gamma_{q}(\alpha)} \int_{0}^{t}(q s / t ; q)_{\alpha-1} d_{q} s+\frac{M T \alpha}{\Gamma_{q}(\alpha+1)} \\
& \leq\left|x_{0}\right|+G+\frac{2 L_{r} T^{\alpha}}{\Gamma_{q}(\alpha+1)}+\frac{M T^{\alpha}}{\Gamma_{q}(\alpha+1)} \\
& \leq \|\left. x_{0}\right|_{C(J, X)}+G+(2 L r+M) \frac{T^{\alpha}}{\Gamma_{q}(\alpha+1)} \leq r
\end{aligned}
$$

By the choice of $L$ and $r$. Now take $x, y \in \mathscr{C}(\mathscr{f}, \mathscr{X})$. Then we get:

$$
\begin{aligned}
& |(P x)(t)-(P y)(t)| \\
& \leq|g(x)-g(y)| \\
& +\frac{t^{\alpha-1}}{\Gamma_{q}(\alpha)} \int_{0}(q s / t ; q)_{\alpha-1}\left|\begin{array}{l}
F(s, x(s), x(\lambda s)) \\
-F(s, y(s), y(\lambda s))
\end{array}\right| d_{q} s \\
& \leq b|x-y| \\
& +\frac{t^{\alpha-1}}{\Gamma_{q}(\alpha)} \int_{0}^{f}(q s / t ; q)_{\alpha-1} L\left(\begin{array}{l}
\max _{s \in X}|x(s)-y(s)| \\
+\max _{s \in Y}|x(\lambda s)-y(\lambda s)|
\end{array}\right) d_{q} s \\
& \leq b|x-y|+\frac{2 L|x-y| t^{\alpha-1}}{\Gamma_{q}(\alpha)} \int_{0}^{f}(q s / t ; q)_{\alpha-1} d_{q} s \\
& \leq b|x-y|+\frac{2 L|x-y| T^{\alpha}}{\Gamma_{q}(\alpha+1)} \\
& \leq\left(b+\frac{2 L T^{\alpha}}{\Gamma_{q}(\alpha+1)}\right)\|x-y\|_{\mathscr{E}(\mathcal{S}, x)}=\Omega_{\mathrm{b}, \mathrm{L}, \mathrm{T}, \alpha, \mathrm{q}}\|x-y\|_{\mathscr{E}(\mathcal{G}, x)}
\end{aligned}
$$

Thus:

$$
\|(P x)-(P y)\|_{\mathscr{E}(\mathcal{f} ; x)} \leq \Omega_{\mathrm{b}, \mathrm{L}, \mathrm{T}, \alpha, \mathrm{q}}\|x-y\|_{\mathscr{E}(\mathscr{Y} ; x)}
$$

where, $\Omega_{b, L, T, \alpha, q}:=\left(b+\frac{2 L T^{\alpha}}{\Gamma_{q}(\alpha+1)}\right)$ depends only on the parameters of the problem and since $\Omega_{b, L, T, \alpha, q}<1$, the result follows in view of the contraction mapping principle due to Chebyshev norm.

\section{Theorem 4.9}

If the hypotheses (A1)-(A3) and (3) are satisfied. Then, the Equation (1) is $U-H-q$-Mittag-Leffler stable.

\section{Proof}

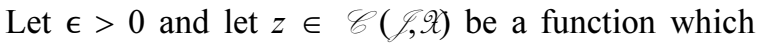
satisfies the inequality:

$\left|{ }^{c} D_{q}^{\alpha} z(t)-F(t, z(t), z(\lambda t))\right| \leq \epsilon e_{\alpha}\left(t^{\alpha} ; q\right)$, for any $t \in \mathscr{f}$

and let $x \in \mathscr{C}(\mathscr{C}, \mathscr{x})$ be the unique solution of the following $q$-fractional order pantograph equation:

$$
\begin{aligned}
& { }^{c} D_{q}^{\alpha} x(t)=F(t, x(t), x(\lambda t)), \quad t \in \mathscr{f}, 0<\alpha<1, \\
& z(0)+g(z)=x_{0}
\end{aligned}
$$

So:

$$
x(t)=x_{0}-g(x)+\frac{1}{\Gamma_{q}(\alpha)} \int_{b}^{(}(t-q s)^{(\alpha-1)} F(s, x(s), x(\lambda s)) d_{q} s
$$

By applying Lemma 4.3, we get: 


$$
\left|\begin{array}{l}
z(t)-x_{0}+g(z) \\
-\frac{1}{\Gamma_{q}(\alpha)} \int_{0}^{t}(t-q s)^{(\alpha-1)} F(s, z(s), z(\lambda s)) d_{q} s
\end{array}\right| \leq \epsilon e_{\alpha}\left(t^{\alpha} ; q\right)
$$

For each $t \in \mathscr{f}$, we have:

$$
\begin{aligned}
& |z(t)-x(t)| \\
& \leq\left|\begin{array}{l}
z(t)-x_{0}+g(z) \\
-\frac{1}{\Gamma_{q}(\alpha)} \int_{0}^{t}(t-q s)^{(\alpha-1)} F(s, z(s), z(\lambda s)) d_{q} s
\end{array}\right| \\
& +\mid g(x)-g(z) \\
& +\frac{1}{\Gamma_{q}(\alpha)} \int_{0}^{(}(t-q s)^{(\alpha-1)}\left(\begin{array}{l}
F(s, z(s), z(\lambda s)) \\
-F(s, x(s), x(\lambda s))
\end{array}\right) d_{q} s \mid \\
& \leq \epsilon e_{\alpha}\left(t^{\alpha} ; q\right)+|g(z)-g(x)| \\
& +\frac{1}{\Gamma_{q}(\alpha)} \int_{0}(t-q s)^{(\alpha-1)} \\
& |F(s, z(s), z(\lambda s))-F(s, x(s), x(\lambda s))| \mid d_{q} s \\
& \leq \epsilon e_{\alpha}\left(t^{\alpha} ; q\right)+b|z(t)-x(x)| \\
& +\frac{1}{\Gamma_{q}(\alpha)} \int_{0}^{(}(t-q s)^{(\alpha-1)}[|z(s)-x(s)|+|z(\lambda s)-x(\lambda s)|] d_{q} s
\end{aligned}
$$

For $u \in \mathscr{C}(\mathscr{C}, \mathscr{X})$ we consider the operator $A: \mathscr{C}(\mathscr{C}, \mathfrak{X})$ $\rightarrow \mathscr{C}(\mathscr{f}, \mathscr{R})$ defined by:

$$
\begin{aligned}
(A u)(t) & =\epsilon e_{\alpha}\left(t^{\alpha} ; q\right)+b u(t) \\
& +\frac{L}{\Gamma_{q}(\alpha)}\left(\begin{array}{c}
\int_{0}^{t}(t-q s)^{(\alpha-1)} u(s) d_{q} s \\
+\int_{0}^{t}(t-q s)^{(\alpha-1)} u(s) d_{q} s
\end{array}\right), t \in \mathcal{J}
\end{aligned}
$$

Next, we verify that $A$ is a Picard operator.

For all $t \in \mathscr{f}$, it follows (A2):

$$
\begin{aligned}
& |(A u)(t)-(A v)(t)| \\
& \leq b|u(t)-v(t)| \\
& +L\left(\frac{t^{\alpha-1}}{\Gamma_{q}(\alpha)} \int_{0}^{L}(q s / t ; q)_{\alpha-1}[|u(s)-v(s)|+|u(\lambda s)-v(\lambda s)|] d_{q} s\right) \\
& \leq b\|u-v\|_{C(J, X)}+\frac{2 L T^{\alpha}}{\Gamma_{q}(\alpha+1)}\|u-v\|_{\mathscr{E}(,, x)} \\
& \leq\left(b+\frac{2 L T^{\alpha}}{\Gamma_{q}(\alpha+1)}\right)\|u-v\| \mathscr{E}(\mathscr{f}, \mathscr{X}) \\
& =\Omega_{b, L, T, \alpha, q}\|u-v\| \mathscr{E}(\quad, \mathscr{X})
\end{aligned}
$$

Thus, $A$ is a contraction via the Chebyshev norm $\|\cdot\|$ on $\mathscr{C}(\mathscr{f}, \mathfrak{X})$ due to $(3)$.
Applying the Banach contraction principle to $A$, we derive that $A$ is a Picard operator and $F_{A}=\left\{u^{*}\right\}$. Then for $t \in \mathcal{F}$.

$$
\begin{aligned}
& u^{*}(t)=\epsilon e_{v}\left(t^{\alpha} ; q\right)+b u^{*}(t) \\
& +\frac{L}{\Gamma_{q}(\alpha)}\left(\begin{array}{l}
\int_{0}^{L}(t-q s)^{(\alpha-1)} u^{*}(s) d_{q} s \\
+\int_{0}^{t}(t-q s)^{(\alpha-1)} u^{*}(\lambda s) d_{q} s
\end{array}\right)
\end{aligned}
$$

It remains to verify that the solution $u^{*}$ is increasing. Indeed, for $0 \leq t_{1}<t_{2} \leq b$ and denote $m: \min _{s \in J}\left[u^{*}(s)+(\lambda s)\right] \in \mathbb{R}_{+}$, we have:

$$
\begin{aligned}
& u^{*}\left(t_{2}\right)-u^{*}\left(t_{1}\right) \\
& =\epsilon\left[e_{\alpha}\left(t_{2}^{\alpha} ; q\right)-e_{\alpha}\left(t_{1}^{\alpha} ; q\right)\right]+b\left[u^{*}\left(t_{2}\right)-u^{*}\left(t_{1}\right)\right] \\
& +\frac{L}{\Gamma_{q}(\alpha)} \int_{0}^{t_{1}}\left[\left(t_{1}-q s\right)^{(\alpha-1)}-\left(t_{2}-q s\right)^{(\alpha-1)}\right] \\
& {\left[u^{*}(s)+u^{*}(\lambda s)\right] d_{q} s} \\
& +\frac{L}{\Gamma_{q}(\alpha)} \int_{l_{1}}^{t_{2}}\left(t_{2}-q s\right)^{(\alpha-1)}\left[u^{*}(s)+u^{*}(\lambda s)\right] d_{q} s \\
& \leq \epsilon\left[e_{\alpha}\left(t_{2}^{\alpha} ; q\right)-e_{\alpha}\left(t_{1}^{\alpha} ; q\right)\right]+b\left[u^{*}\left(t_{2}\right)-u^{*}\left(t_{1}\right)\right] \\
& +\frac{m L}{\Gamma_{q}(\alpha)} \int_{0}^{f_{1}}\left[t_{2}^{\alpha-1}\left(q s / t_{2} ; q\right)_{\alpha-1}-t_{1}^{\alpha-1}\left(q s / t_{1} ; q\right)_{\alpha-1}\right] d_{q} s \\
& +\frac{m L}{\Gamma_{q}(\alpha)} \int_{1}^{\gamma_{1}^{2}} t_{2}^{\alpha-1}\left(q s / t_{2} ; q\right)_{\alpha-1} d_{q} s \\
& =\epsilon\left[e_{\alpha}\left(t_{2}^{\alpha} ; q\right)-e_{\alpha}\left(t_{1}^{\alpha} ; q\right)\right]+b\left[u^{*}\left(t_{2}\right)-u^{*}\left(t_{1}\right)\right] \\
& +\frac{m L}{\Gamma_{q}(\alpha+1)}\left(t_{2}^{\alpha}-t_{1}^{\alpha}\right)>0
\end{aligned}
$$

Then, we obtain $u^{*}$ is increasing:

$$
u^{*} \leq \epsilon e_{\alpha}\left(t^{\alpha} ; q\right)+b u^{*}+\frac{2 L}{\Gamma_{q}(\alpha)} \int_{0}^{(}(t-q s)^{(\alpha-1)} u^{*}(s) d_{q} s
$$

Using Lemma 4.6 and Remark 4, we get:

$$
\begin{aligned}
& u^{*}(t) \leq \frac{\epsilon e_{\alpha}\left(t^{\alpha} ; q\right)}{(1-b)} \\
& +\frac{1}{(1-b)} \cdot \frac{2 L}{\Gamma_{q}(\alpha)} \int_{0}^{t}(t-q s)^{(\alpha-1)} u^{*}(s) d_{q} s \\
& u^{*}(t) \leq C_{e_{\alpha}} \epsilon e_{\alpha}\left(t^{\alpha} ; q\right)
\end{aligned}
$$

where, $C_{e_{\alpha}}:=\frac{1}{1-b} \cdot e_{\alpha}\left(\frac{2 L}{1-b} T^{\alpha} ; q\right)$. 
In particular, if $u=|z-x|$, from (6), $u \leq A u$ and applying the Lemma 4.5 we obtain $u \leq u^{*}$, where $A$ is a picard and increasing operator. As a result, we know:

$$
|z(t)-x(t)| \leq C_{e_{\alpha}} \epsilon e_{\alpha}\left(t^{\alpha} ; q\right)
$$

Thus, the Equation (1) is $U-H-q$-Mittag-Leffler stable.

\section{Remark 4.10}

Theorem 3.1 and 4.9 can easily be extended to the generalized $q$-fractional multi-pantograph of the form:

$$
\begin{aligned}
& { }^{c} D_{q}^{\alpha} x(t)=F\left(t, x(t), x\left(\lambda_{1} t\right), \ldots, x\right), \quad 0<q>1, t \in[0, T] \\
& x(0)+g(x)=x_{0}
\end{aligned}
$$

where, ${ }^{c} D_{q}^{\alpha}$ is the Caputo $q$-fractional derivative, $\alpha \in$ $(0,1)$.

Now we give an example to illustrate our results.

\section{An Example}

Consider the nonlinear $q$-fractional pantograph problem given by:

$$
\left\{\begin{array}{l}
{ }^{c} D_{q}^{\alpha} x(t)=\frac{1}{5}+\frac{1}{10} x(t) \frac{1}{10} x\left(\frac{t}{2}\right), t \in[0,1], \\
x(0)+\sum_{i=1}^{m} a_{i} x\left(t_{i}\right)=0,0<t_{1}<t_{2}<\cdots<t_{m}<1
\end{array}\right.
$$

where, $\alpha \in(0,1), q \in(0,1), a_{i}>0, i=0,1,2, \ldots, m$ are positive constants with:

$$
\sum_{i=1}^{m} a_{i} \leq \frac{1}{3}
$$

Set:

$$
F(t, u, v)=\frac{1}{5}+\frac{1}{10} u+\frac{1}{10} v, t \in[0,1], u, v \in \mathscr{X}
$$

and:

$$
g(x)=\sum_{i=1}^{m} a_{i} x\left(t_{i}\right) .
$$

Let $u, v, \bar{u}, \bar{v} \in \mathscr{X}$ and $t \in[0,1]$. Then we have:

$$
|F(t, u, v)-F(t, \bar{u}, \bar{v})| \leq \frac{1}{10}(|u-\bar{u}|+|v-\bar{v}|)
$$

On the other hand, we have:

$$
\begin{aligned}
& |g(u)-g(\bar{u})| \leq\left|\sum_{i=1}^{m} a_{i} u-\sum_{i=1}^{m} a_{i} \bar{u}\right| \\
& \leq \sum_{i=1}^{m} a_{i}|u-\bar{u}| \\
& \leq \frac{1}{3}|u(t)-\bar{u}(t)|
\end{aligned}
$$

Denote: $\alpha=\frac{1}{8}, L=\frac{1}{10}, q=\frac{1}{2}$ and $b=\frac{1}{3}$.

Thus:

$$
L \leq \frac{\Gamma_{q}(\alpha+1)}{2} \Leftrightarrow \Gamma_{q}(\alpha+1) \geq 2 L=0.2
$$

where, $\frac{\Gamma_{q}(\alpha+1)}{2}=\frac{0.957935}{2}=0.4789675$.

Equation (7) follows the inequality:

$$
\left|{ }^{c} D_{q}^{\alpha} z(t)-F(t, x(t), x(\lambda t))\right| \leq \epsilon e_{\alpha}\left(t^{\frac{1}{8}} ; \frac{1}{2}\right)
$$

Now all assumptions in Theorem 3.1 and 4.9 are satisfied, the problem (7) has a unique solution and the Equation (7) is $U-H-q$-Mittag-Leffler stable with:

$$
|z(t)-x(t)| \leq C_{e_{\alpha}} \epsilon e_{\alpha}\left(t^{\frac{1}{8}} ; \frac{1}{2}\right)
$$

\section{Acknowledgement}

The authors would like to thank the reviewers for their constructive comments and suggestions.

\section{Author's Contributions}

The paper was realized in complete collaboration. All authors have read and approved the final manuscript.

\section{Ethics}

The Authors declare there is not conflict of interest.

\section{References}

Agarwal, R.P., B. Ahmad, A. Alsaedi and H. Al-Hutami, 2014. On nonlinear fractional $q$-difference equations involving two fractional orders with threepoint nonlocal boundary conditions. Dynam. Contin. Discrete Impuls. Syst., 21: 135-151.

Al-Yami, M., 2016. A cauchy problem for some fractional $q$-difference equations with nonlocal conditions. Am. J. Math., 6: 159-165.

Andras, S. and J.J. Kolumban, 2013. On the Ulam-Hyers stability of rst order differential systems with nonlocal initial conditions. Nonlinear Anal. Theory Meth. Applied, 82: 1-11. 
Balachandran, K., S. Kiruthika and J.J. Trujillo, 2013. Existence of solutions of nonlinear fractional pantograph equations, Acta Math. Sci., 33: 1-9.

Bashir, A. and S. Sivasundaram, 2008. Some existence results for fractional integro-differential equations with nonlocal conditions. Commun. Applied Anal., 12: 107-112.

Hassan, H.A., 2016. Generalized $q$-Taylor formulas. Adv. Differ. Eq., 162: 1-12. DOI: $10.1186 / \mathrm{s} 13662-016-0894-3$.

Hilfer, R., 1999. Application of Fractional Calculus in Physics. 1st Edn., World Scientic, Singapore.

Hyers, D.H., G. Isac and T.M. Rassias, 1998. Stability of functional equations in several variables. Progr. Nonlinear Differential Equations Appl., Boston (MA): Birkhauser.

Ibrahim, R.W., 2012. Generalized Ulam-Hyers stability for fractional differential equations. Int. J. Math., 23: 1-9. DOI: 10.1142/S0129167X12500565

Jackson, F., 1908. On $q$-functions and a certain difference operator. Trans. R. Society Edinburgh, 46: $253-281$.

Jackson, F., 1910. On $q$-denite integrals. Q. J. Mech. Applied Math., 41: 193-203.

Jung, S.M., 2004. Hyers-Ulam stability of linear differential equations of rst order. Applied Math. Lett., 17: 1135-1140.

Koca, I., 2015. A method for solving differential equations of q-fractional order. Applied Mathematics Computation, 266: 1-5.

Lakshmikantham, V., 1994. Theory of Differential Equations with Unbounded Delay. 1st Edn., Kluwer Academic Publications, Dordrecht.
Liang, S. And J. Zhang, 2012. Existence and uniqueness of positive solutions for three-point boundary value problem with fractional $q$-differences. J. Applied Math. Comput., 40: 277-288.

Liu, M.Z. and D. Li, 2004. Runge-Kutta methods for the multi-pantograph delay equation. Applied Math. Comput., 155: 853-871.

Muniyappan, P. and S. Rajan, 2015. Hyers-UlamRassias stability of fractional differential equation. Int. J. Pure Applied Math., 102: 631-642.

Otrocol, D. and V. Ilea, 2013. Ulam stability for a delay differential equation. Cent. Eur. J. Math., 11: 1296-1303.

Podlubny, I., 1999. Fractional Differential Equations. 1st Edn., Academic Press, San Diego.

Stankovic, M.S., P.M. Rajkovic and S.D. Marinkovic, 2009. On $q$-fractional derivatives of riemannliouville and caputo type.

Wang, J. and Y. Zhou, 2012. New concepts and results in stability of fractional differential equations. Commun. Nonlinear Sci. Numer. Simul., 17: 2530-2538.

Wang, J., L. Lv and Y. Zhou, 2011. Ulam stability and data dependence for fractional differential equations with Caputo derivative. Electron. J. Qual. Theory Differ. Eq., 63: 1-10.

Wang, J.R. and Y. Zhang, 2014. Ulam-Hyers-mittagleffler stability of fractional order delay dif-ferential equations. Optimization: A J. Math. Program. Operat. Res., 63: 1181-1190. 